\title{
VARIAÇÃO POR UM ANO DA RADIAÇÃO FOTOSSINTETICAMENTE ATIVA, ULTRAVIOLETA-A E ULTRAVIOLETA-B NO BAIRRO TRINDADE, FLORIANÓPOLIS, SANTA CATARINA
}

Débora Tomazi Pereira ${ }^{1}$ Zenilda Laurita Bouzon² Carmen Simioni ${ }^{3}$

Resumo: O objetivo deste estudo foi medir a intensidade da radiação fotossinteticamente ativa (PAR), radiação ultravioleta $A$ (RUVA) e radiação ultravioleta B (RUVB) durante um ano (maio a dezembro de 2013 e janeiro a abril de 2014) em Florianópolis. As medições ocorreram todas as segundas, quartas e sextas-feiras em intervalos de $1 \mathrm{~h}$, no período das 10:00 h às 14:00 $\mathrm{h}$. As maiores intensidades de PAR foram observadas em janeiro, seguidas de fevereiro, novembro e dezembro. As maiores intensidades de RUVA ocorreram de setembro a fevereiro, e para RUVB, de novembro a fevereiro. Tendo em conta o horário, as intensidades de PAR não mostraram diferença estatística, as intensidades de RUVA foram maiores que as de RUVB durante todos os meses e em todos os momentos. Para RUVA, os picos de intensidade ocorreram ao meio-dia, enquanto o RUVB foi mais constante durante as horas medidas. Sazonalmente, observou-se que os índices mais altos de PAR, RUVA e RUVB ocorreram no verão.

Palavras-chave: Brasil. Irradiância. Medições.Radiação Ultravioleta.

\section{VARIATION FOR A YEAR OF PHOTOSYNTHETICALLY ACTIVE, ULTRAVIOLET- A AND ULTRAVIOLET-B RADIATION IN THE NEIGHBORHOOD TRINDADE, FLORIANÓPOLIS, SANTA CATARINA}

Abstract: The objective of this study was to measure the intensity of photosynthetically active radiation (PAR), ultraviolet $A$ (UVAR) and ultraviolet $B$ (UVBR) for one year (May to December 2013 and January to April 2014) in Florianópolis. Measurements were performed every Monday, Wednesday and Friday at 1-hour intervals from 10:00 a.m. to 2:00 p.m. The highest intensities of PAR were observed in January, followed by February, November and December. The highest intensities of UVAR occurred from September to February, and for UVBR, from November to February. Taking into account the time, the incidences of PAR showed no statistical difference, UVAR intensities were higher than those of UVBR during all months and at all times. For UVAR the incidence peaks occur at noon, while the UVBR was more constant during measured times. Seasonally, it was noted that the highest indices of PAR, UVAR and UVBR occurred in summer.

Keywords: Brazil. Irradiance. Measurements. Ultraviolet Radiation.

\footnotetext{
${ }^{1}$ Universidade Federal de Santa Catarina, Departamento de Biologia Celular, Embriologia e Genética, Florianópolis, Brasil, de.tomazi@gmail.com, https://orcid.org/0000-0002-2330-8812

${ }_{2}^{2}$ Universidade Federal de Santa Catarina, Departamento de Biologia Celular, Embriologia e Genética, Florianópolis, Brasil, zenilda.bouzon@ufsc.br, https://orcid.org/0000-0001-9472-1260

${ }^{3}$ Universidade Federal de Santa Catarina, Departamento de Biologia Celular, Embriologia e Genética, Florianópolis, Brasil, carmensimioni@hotmail.com, https://orcid.org/0000-0001-5629-9361
} 


\section{VARIACIÓN POR UN AÑO DE RADIACIÓN FOTOSINTÉTICAMENTE ACTIVA, ULTRAVIOLETA-A Y ULTRAVIOLETA-B EN EL BARRIO TRINDADE, FLORIANÓPOLIS, SANTA CATARINA}

Resumen: El objetivo de este estudio fue medir la intensidad de la radiación fotosensídicamente activa (PAR), radiación ultravioleta $A$ (RUVA) y radiación ultravioleta B (RUVB) durante un año (mayo a diciembre de 2013 y enero a abril de 2014) en Florianópolis. Las mediciones se realizaron todos los lunes, miércoles y viernes a intervalos de $1 \mathrm{~h}$, en el período de las 10:00 a las 14:00 h. Las mayores intensidades de PAR se observaron en enero, seguidas de febrero, noviembre y diciembre. Las mayores intensidades de RUVA ocurrieron de septiembre a febrero, y para RUVB, de noviembre a febrero. Teniendo en cuenta el horario, las incidencias de PAR no mostraron diferencia estadística, las intensidades de RUVA fueron mayores que las de RUVB durante todos los meses y en todo momento. Para RUVA, los picos de incidencia ocurrieron al mediodía, mientras que el RUVB fue más constante durante las horas medidas. Sazonalmente, se observó que los índices más altos de PAR, RUVA y RUVB ocurrieron en el verano.

Palabras clave: Brasil. Irradiancia. Mediciones. Radiación Ultravioleta.

\section{Introdução}

A luz solar é de grande importância para a manutenção da vida no planeta Terra (DIFFEY, 1991) e é dividida, de acordo com o comprimento de onda dentro do espectro eletromagnético, em: raios gama, raios $x$, radiação ultravioleta (RUV), radiação fotossinteticamente ativa (PAR), infravermelho, micro-ondas e ondas de rádio. Raios gama, raios $x$ e parte da RUV, chamados de radiação ionizante, não atingem a superfície terrestre, enquanto que a outra parte da RUV, PAR, infravermelho, micro-ondas e ondas de rádio, chamados de radiação não-ionizante atingem a superfície do nosso planeta (ANDERSON et al., 2002; DIFFEY, 2002; BALOGH et al., 2011). Dentro destas radiações que atingem a Terra, o comprimento de onda com maior efeito deletério para os organismos é a RUV.

A RUV é dividida, de acordo com o II Congresso Internacional sobre a Luz de 1932 em Copenhagen, em três tipos: ultravioleta-A (RUVA), ultravioleta-B (RUVB) e ultravioleta-C (RUVC), sendo que a RUVA compreende de 400-315 nm, a RUVB de 315-280 nm e a RUVC de 280-100 nm (DIFFEY, 2002). A quantidade e a qualidade da RUV que chega à superfície terrestre dependem das propriedades da atmosfera (DIFFEY, 1991), e a maior interação entre RUV e atmosfera ocorre na estratosfera, camada onde se encontra o ozônio $\left(\mathrm{O}_{3}\right)$ e moléculas de $\mathrm{N}_{2}$ e $\mathrm{O}_{2}$, ocorrendo assim atenuação da radiação (DIFFEY, 2002). Além disso, as moléculas de água presentes nas nuvens também espalham a RUVA e RUVB (DIFFEY, 2002).

$\mathrm{O}_{3}$ é um dos gases mais representativos e reativos quando se fala em química da atmosfera, podendo interagir com substâncias químicas que chegam à 
atmosfera (KIRCHOFF, 1988). As moléculas de $\mathrm{O}_{3}$ são formadas e dissociadas constantemente na estratosfera (CASSETI; MACEDO, 1990; OKUNO et al., 1996). Primeiramente, comprimentos de ondas abaixo de $290 \mathrm{~nm}$ (RUVC, raios $x$ e raios gama) não chegam à superfície terrestre, com exceção de altas altitudes, pois são atenuados por moléculas de oxigênio $\left(\mathrm{O}_{2}\right)$, produzidas por seres fotossintetizantes (DIFFEY, 1991; 2002). As energias da RUVC, do raio x e do raio gama quebram o $\mathrm{O}_{2}$, formando dois átomos de oxigênio $(\mathrm{O})$. Estes átomos podem ligar-se a outras $\mathrm{O}_{2}$ presentes na atmosfera formando $0 \mathrm{O}_{3} \mathrm{O}_{3} \mathrm{O}_{3}$, por sua vez, absorve a energia da RUVB quebrando-se em um $\mathrm{O}_{2}$ e $\mathrm{O}$. $\mathrm{O}$ átomo de oxigênio é instável e pode sequestrar um $\mathrm{O}$ da molécula de $\mathrm{O}_{3}$, formando dois $\mathrm{O}_{2}$, uma molécula estável. Portanto, $3 \mathrm{O}_{2}$ formam $2 \mathrm{O}_{3}$, através da presença de radiação ionizante; e $2 \mathrm{O}_{3}$ formam $3 \mathrm{O}_{2}$ através da RUVB, seguindo assim o ciclo do $\mathrm{O}_{2} / \mathrm{O}_{3}$ (KIRCHOFF, 1988). Assim, temos que a radiação RUVA, infravermelho, micro-ondas e ondas de rádio, ondas com maior comprimento de onda, porém menor energia, são fracamente atenuadas pelo $\mathrm{O}_{3}$, chegando em grandes quantidades à superfície terrestre; a radiação RUVB é atenuada de maneira eficiente, mas não completamente pelo $\mathrm{O}_{3}$; e a radiação RUVC é completamente atenuada pelo $\mathrm{O}_{2}$ (BEISSERT; GRANSTEIN, 1996; DE LAAT et al., 1996; SCHARFFETTER-KOCHANEK et al., 1997).

Até $\mathrm{o}$ início da década de 70 , não se imaginava que a atividade humana poderia levar à destruição do $\mathrm{O}_{3}$ (MOLINA; ROWLAND, 1974; STOLARSKI; CICERONE, 1974). Pesquisas nesta área despontaram, até que, em 1973, três cientistas, Paul Josef Crutzen, Frank Sherwood Rowland e Mario Molina, verificaram os efeitos dos clorofluorcarbonetos (CFCs) e bromofluorocarbonos (BrFCs), classes de gases que podem ficar na sua forma estável por anos na atmosfera, e chegar à estratosfera (MOLINA; ROWLAND, 1974). Na estratosfera, estes gases podem ser dissociados por energia proveniente da radiação ionizante e liberar o elemento cloro, flúor e bromo, os quais se ligam ao $\mathrm{O}$ e $\mathrm{O}_{2}$, dissociando a molécula de $\mathrm{O}_{3}$ estratosférico (MOLINA; MOLINA, 1987; STOLARSKI; CICERONE, 1974). Esta problemática nos leva ao índice no qual reduzir $1 \%$ da camada de $\mathrm{O}_{3}$, aumentam-se de $1,3 \%$ a $1,8 \%$ os níveis de RUVB que atingem a superfície do planeta (HOLLÓSY, 2001).

De acordo com o último relatório do Intergovernmental Panel on Climate Change (IPPC) de 2013 a concentração dos gases de efeito estufa na atmosfera é a mais alta desde os últimos 800 mil anos, e se nenhuma providência for tomada, a camada de ozônio será reduzida, facilitando a passagem de radiações, a qual fará 
com que o planeta esquente, elevando a temperatura média global em $5{ }^{\circ} \mathrm{C}$ e os níveis dos oceanos devem aumentar 0,7 metros até 2100 (IPCC, 2013).

De acordo com o Instituto Nacional de Pesquisas Espaciais (INPE), a região fitogeográfica Temperada Quente, a qual compreende o Estado de Santa Catarina, no verão, recebe RUVB de 9 a 14 na escala de índice de RUVB, a qual vai de 0 a 16, evidenciando que a região recebendo altas doses de RUVB no verão. Porém, faltam informações das reais medidas de RUV no Estado de Santa Catarina, principalmente na capital, Florianópolis, um município que cresceu muito nos últimos anos e junto com este crescimento vem a poluição, podendo danificar a camada de $\mathrm{O}_{3}$. O conhecimento das intensidades atuais de PAR, RUVA e RUVB no ambiente pode ajudar na compreensão de como o meio ambiente está reagindo ao crescimento de Florianópolis, se algum período anual a cidade sente menos estes aumentos, e também em estudos futuros sobre os efeitos biológicos in situ dessas radiações.

\section{Materiais e Métodos}

\section{Método de Medida das Radiações}

Com a finalidade de atualizar e tornar público as intensidades das radiações fotossinteticamente ativa (PAR), ultravioleta-A (RUVA) e ultravioleta-B (RUVB) da cidade de Florianópolis, mais precisamente do bairro Trindade, foram realizadas medidas destas três radiações nos períodos de maio a dezembro do ano de $2013 \mathrm{e}$ de janeiro a abril do ano 2014, na Universidade Federal de Santa Catarina (UFSC) em Florianópolis, Santa Catarina, Brasil. As medidas ocorreram sempre no mesmo ponto (3 metros acima do nível do mar), no chão de um terreno aberto, ao lado do Centro de Ciências Biológicas (CCB), local sem interferência de sombras exercidas por árvores e/ou prédios. As medidas ocorreram todas as segundas, quartas e sextas-feiras em intervalos de $1 \mathrm{~h}$, no período das 10:00 $\mathrm{h}$ às 14:00 $\mathrm{h}$ com o equipamento Light Radiometer Model PMA 2132 (PAR), PMA 2107B-UW (RUVA+RUVB) e PMA 2102 (RUVA), usando um sensor esférico PMA 2100 sensor (Newburyport, MA, USA). Os sensores eram deixados no local de medida até estabilizarem (em média de 2 minutos). As três radiações foram medidas em $\mathrm{m} / \mathrm{m}^{2}$. Para separação das estações, os meses foram divididos da seguinte forma: de janeiro a março (verão), de abril a junho (outono), de julho a setembro (inverno) e de outubro a dezembro (primavera). 


\section{Análises Estatísticas}

Os dados passaram pelo teste de normalidade de Shapiro-Wilk e todas as amostras apresentaram distribuição normal. Posteriormente, os dados foram analisados por Análise Unifatorial de Variância (ANOVA) com o fator independente sendo as horas, seguido pelo teste a posteriori de Tukey para verificar a significância das diferenças $(p \leq 0,05)$.

\section{Resultados}

\section{Medidas Mensais}

As maiores intensidades de PAR foram observadas em janeiro, seguido de fevereiro, novembro e dezembro, enquanto as intensidades mais baixas foram verificadas em abril (Fig. 1). Quanto à RUVA, as maiores intensidades foram nos meses de setembro a fevereiro, e para a RUVB as maiores intensidades ocorreram nos meses de novembro a fevereiro (Fig. 2).

Levando-se em conta o tempo, as intensidades de PAR não mostraram diferenças estatísticas dentre todos os horários de cada mês (Fig. 3). As intensidades de RUVA foram maiores do que as de RUVB durante todos os meses e em todos os horários (Fig. 4). Para RUVA, os picos de intensidade ocorreram ao meio-dia (Fig. 4), enquanto a intensidade de RUVB é mais constante durante os horários medidos (Fig. 4). 


\section{Medidas Mensais}

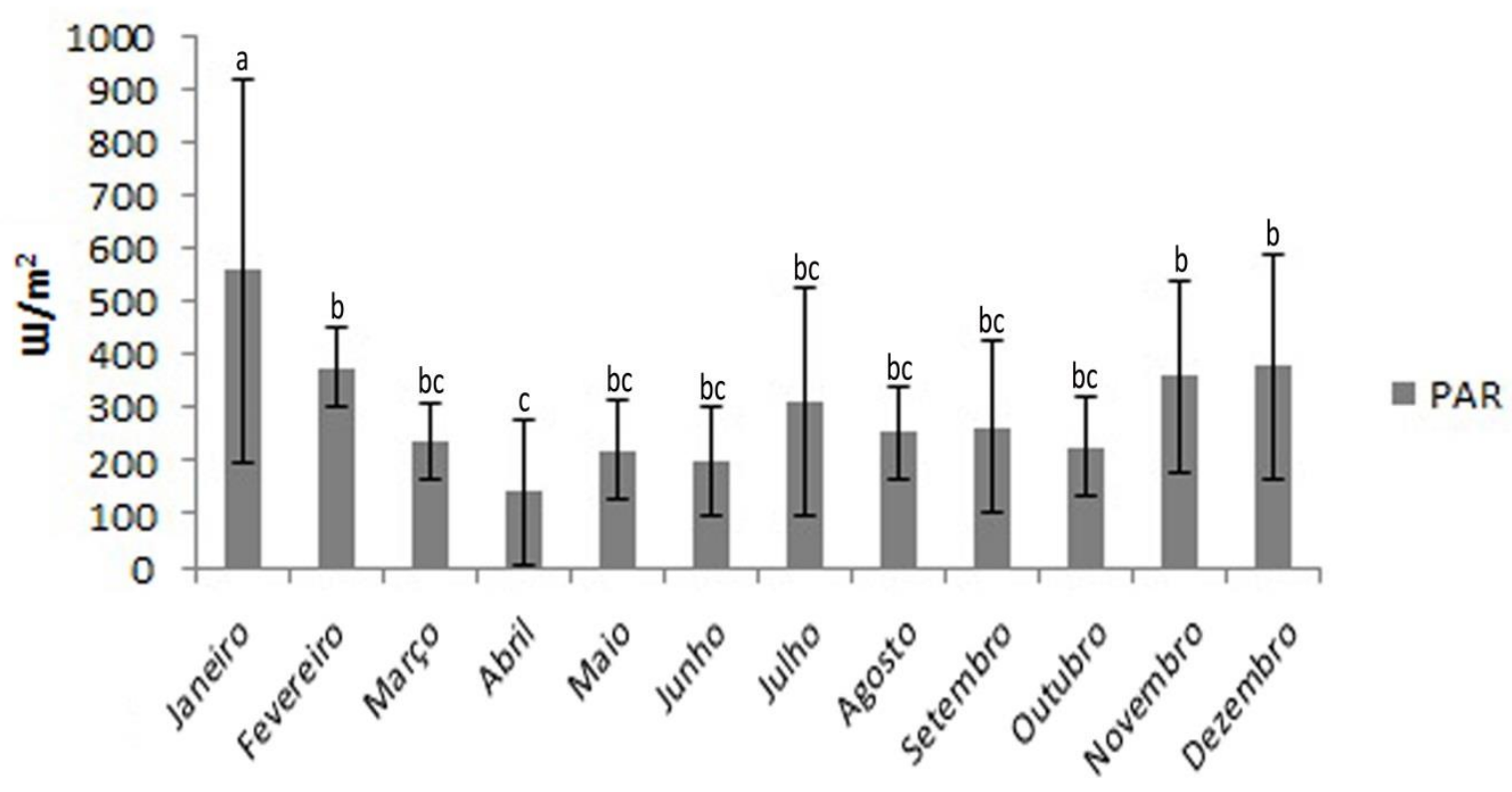

Fig. 1 Medidas mensais de intensidade PAR $\left(\mathrm{U} / \mathrm{m}^{2}\right)$ durante o ano, com maiores intensidades nos meses de janeiro, seguido de fevereiro, novembro e dezembro, enquanto as intensidades mais baixas foram verificadas no mês de abril.

\section{Medidas Mensais}

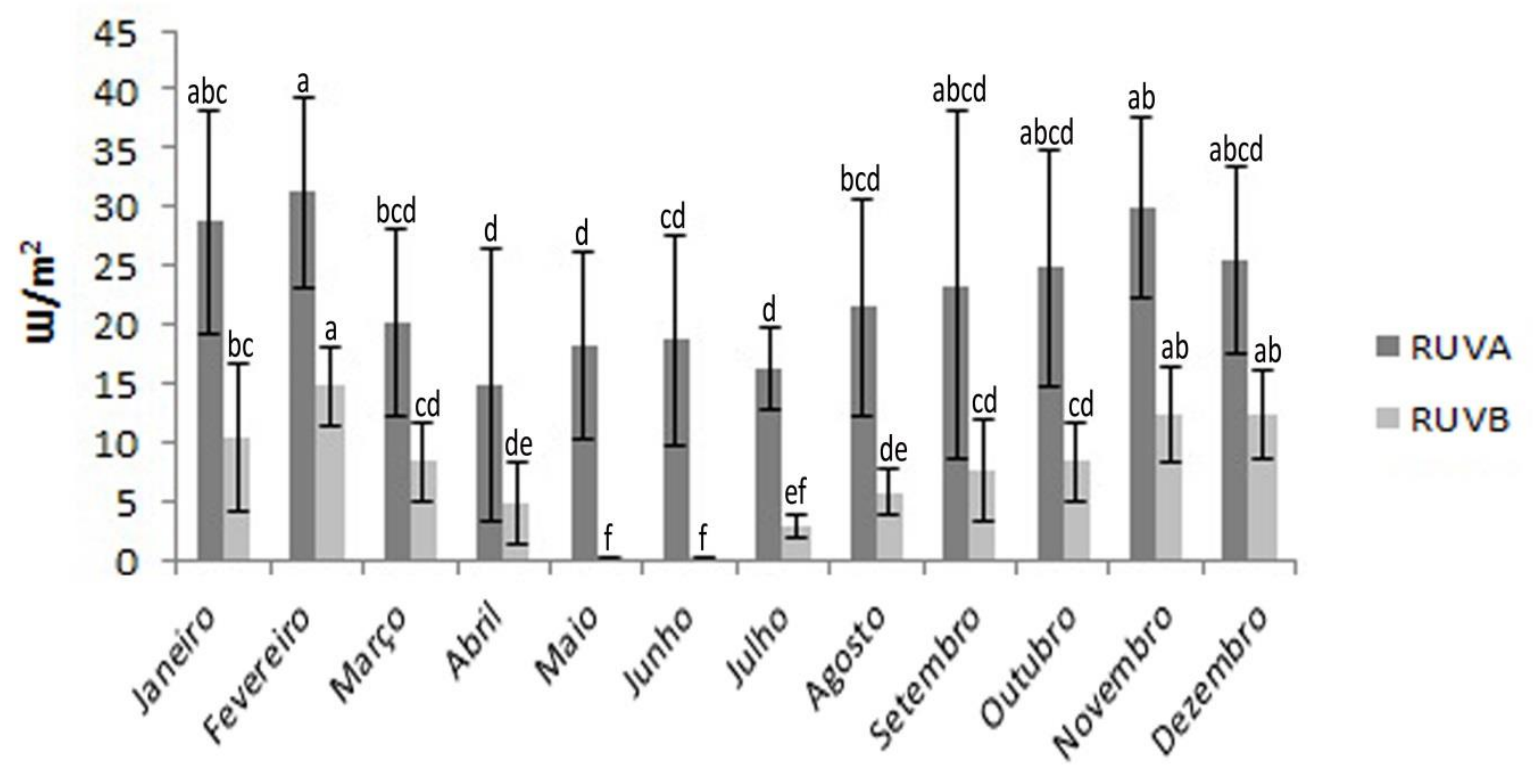

Fig. 2 Medidas mensais de intensidade de RUVA e RUVB $\left(\mathrm{m} / \mathrm{m}^{2}\right)$ durante 0 ano. Maiores intensidades de RUVA foram visualizadas nos meses de setembro a fevereiro, enquanto para a RUVB foram nos meses de novembro a fevereiro. 

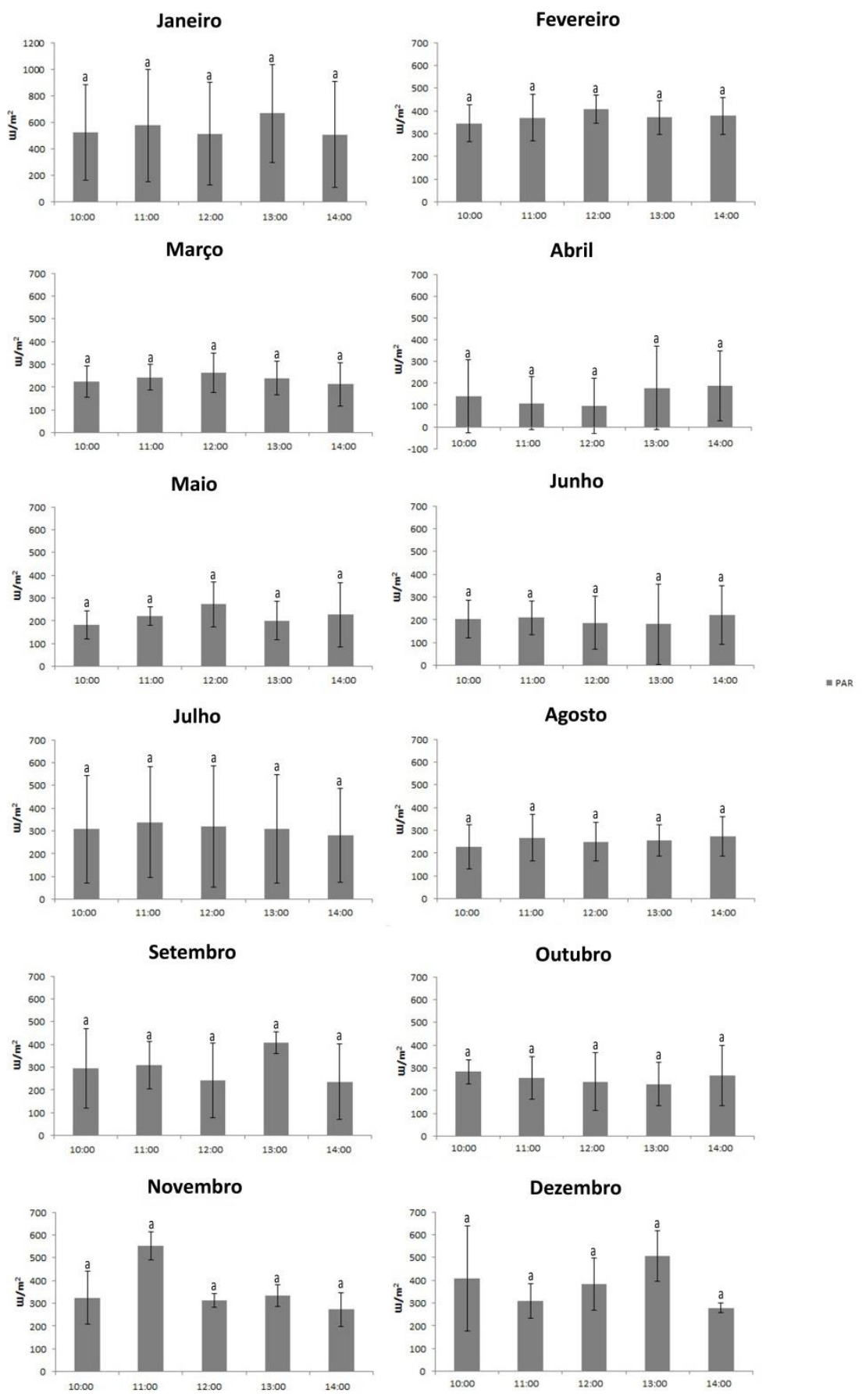

Fig. 3 Intensidade de PAR $\left(\mathrm{W} / \mathrm{m}^{2}\right)$ durante os meses do ano e horário (10:00, 11:00, $12: 00,13: 00$ e 14:00), não apresentando diferenças estatísticas dentre todos os horários dentro de cada mês. 


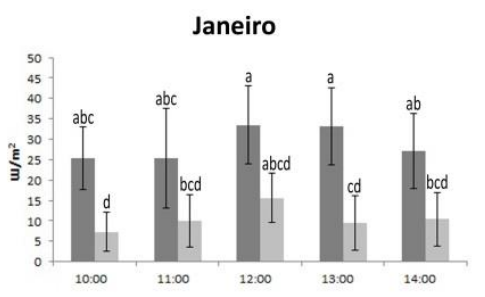

Março

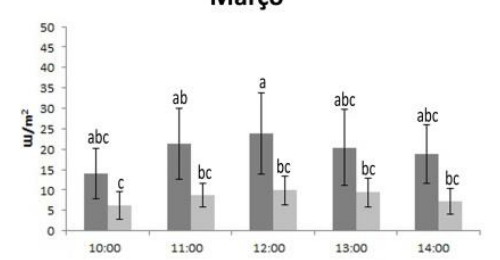

Maio

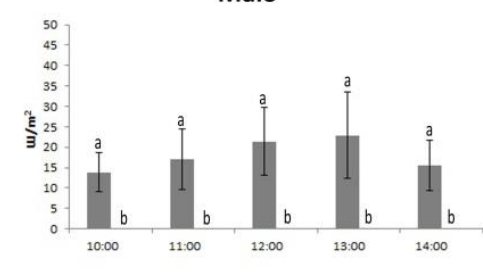

Julho

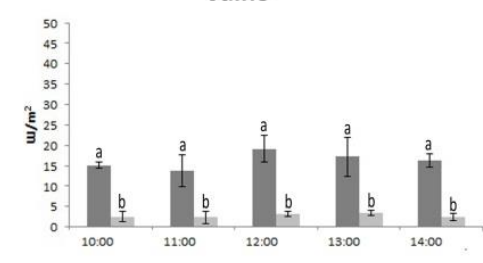

Setembro

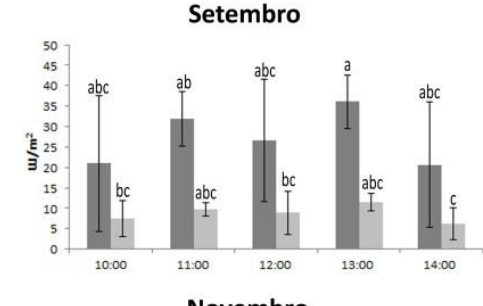

Novembro

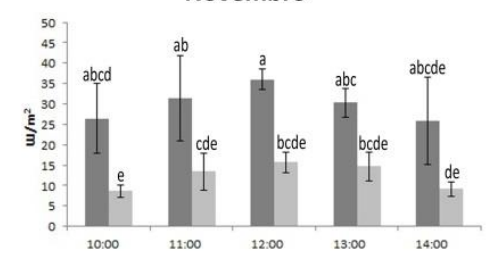

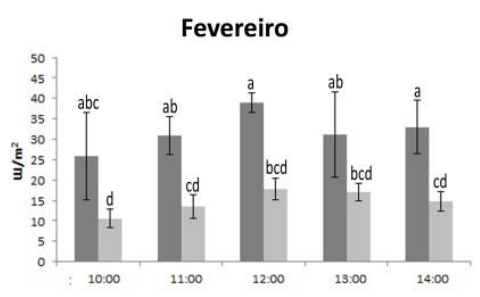

Abril

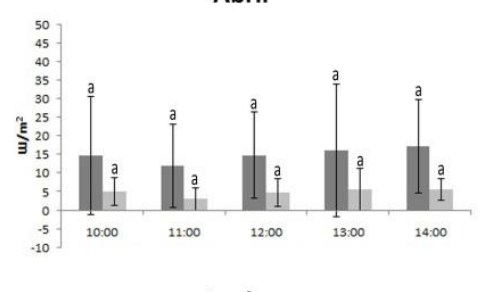

Junho

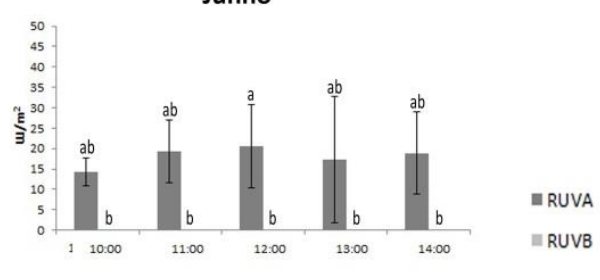

Agosto

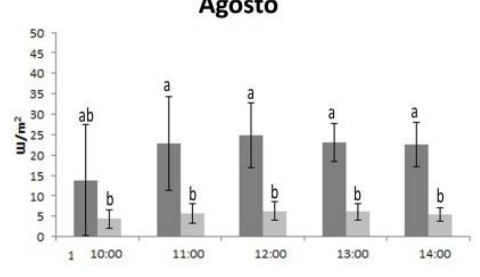

Outubro

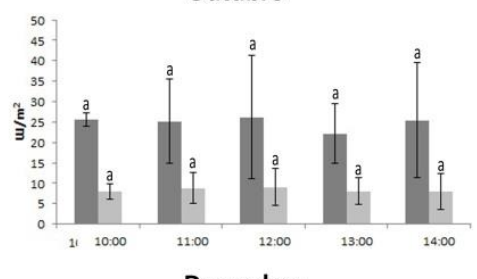

Dezembro

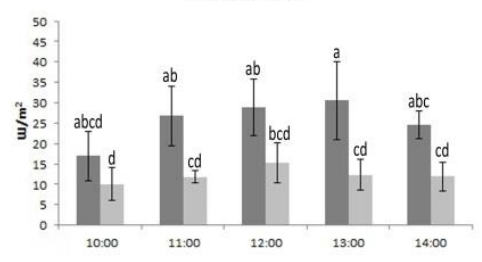

Fig. 4 Intensidades de RUVA e RUVB $\left(\mathrm{W} / \mathrm{m}^{2}\right)$ durante os meses do ano e horário (10:00, 11:00, 12:00, 13:00 e 14:00). Maiores intensidades de RUVA ocorreram ao meio-dia, enquanto a RUVB segue mais constante durante os horários. Intensidades de RUVA foram maiores do que as de RUVB durante todos os meses e em todos os horários.

\section{Medidas Sazonais}

Levando-se em conta as estações, verificou-se que os índices mais altos de PAR ocorreram no verão, seguido da primavera (Fig. 5). O outono foi a estação com a PAR mais baixa, com uma média de $187,47 \mathrm{~m} / \mathrm{m}^{2}$, contra uma média de 287,74 
$\mathrm{U} / \mathrm{m}^{2}$ no inverno. Este mesmo padrão foi repetido para RUVA e RUVB (Fig. 6 ), onde a RUVA média no verão é de $26,83 \mathrm{~m} / \mathrm{m}^{2}$, contra $26,80 \mathrm{~m} / \mathrm{m}^{2}, 17,02 \mathrm{~m} / \mathrm{m}^{2}$ e 21,64 $\mathrm{U} / \mathrm{m}^{2}$ na primavera, outono e inverno, respectivamente. Para a RUVB, a intensidade foi de $11,18 \mathrm{~m} / \mathrm{m}^{2}$ no verão, contra $10,99 \mathrm{~m} / \mathrm{m}^{2}, 1,60 \mathrm{~m} / \mathrm{m}^{2}$ e $5,68 \mathrm{~m} / \mathrm{m}^{2}$ na primavera, outono e inverno, respectivamente. Para PAR não houve diferença estatística entre os horários medidos de cada estação (Fig. 7). Para a RUVA, no outono, inverno e primavera, também não houve diferença estatística entre as intensidades durante os horários medidos, mas no verão, a maior intensidade de RUVA foi observada ao meio-dia (Fig. 8). A RUVB seguiu o mesmo padrão observado em RUVA (Fig. 8).

\section{Medidas Sazonais}

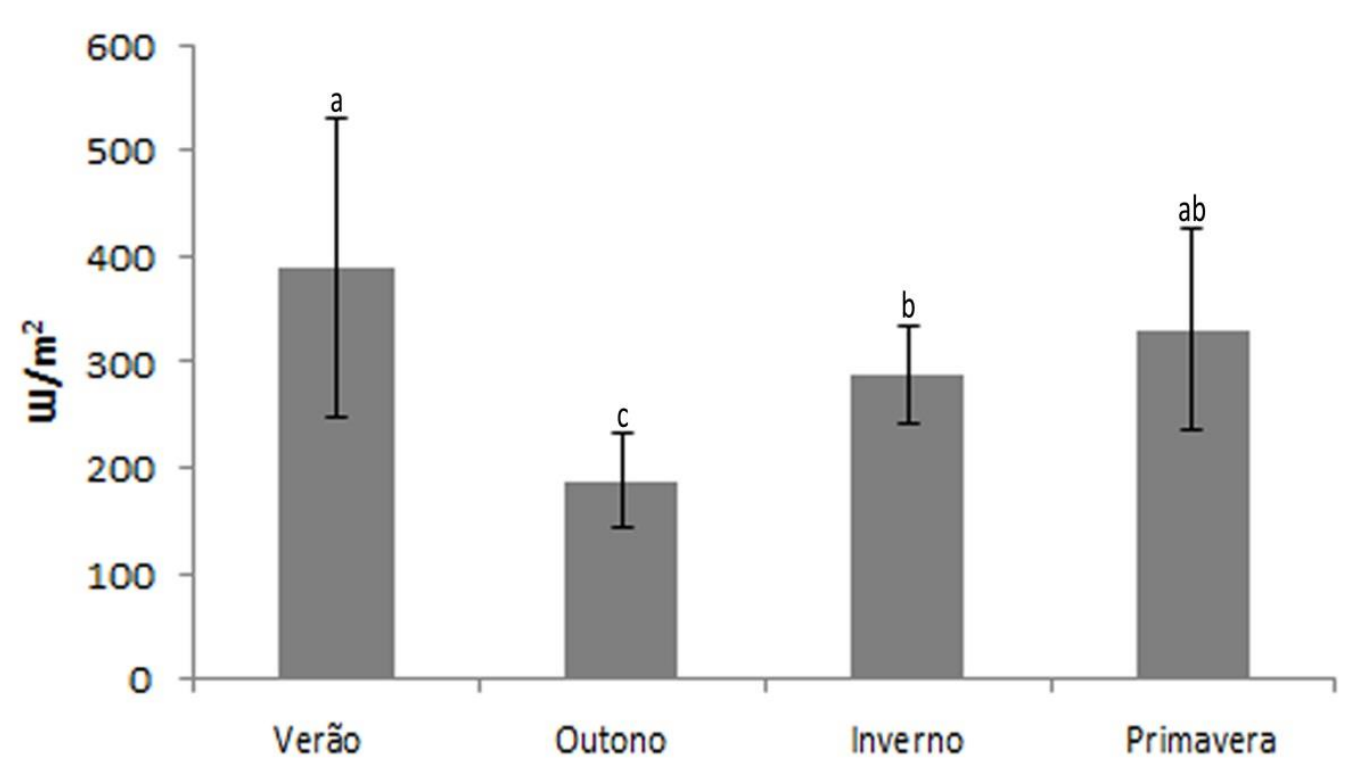

Fig. 5 Medidas sazonais da intensidade de PAR $\left(\mathrm{U} / \mathrm{m}^{2}\right)$. Maiores índices de PAR ocorreram no verão, seguido da primavera, enquanto o outono foi a estação com a PAR mais baixa. 


\section{Medidas Sazonais}

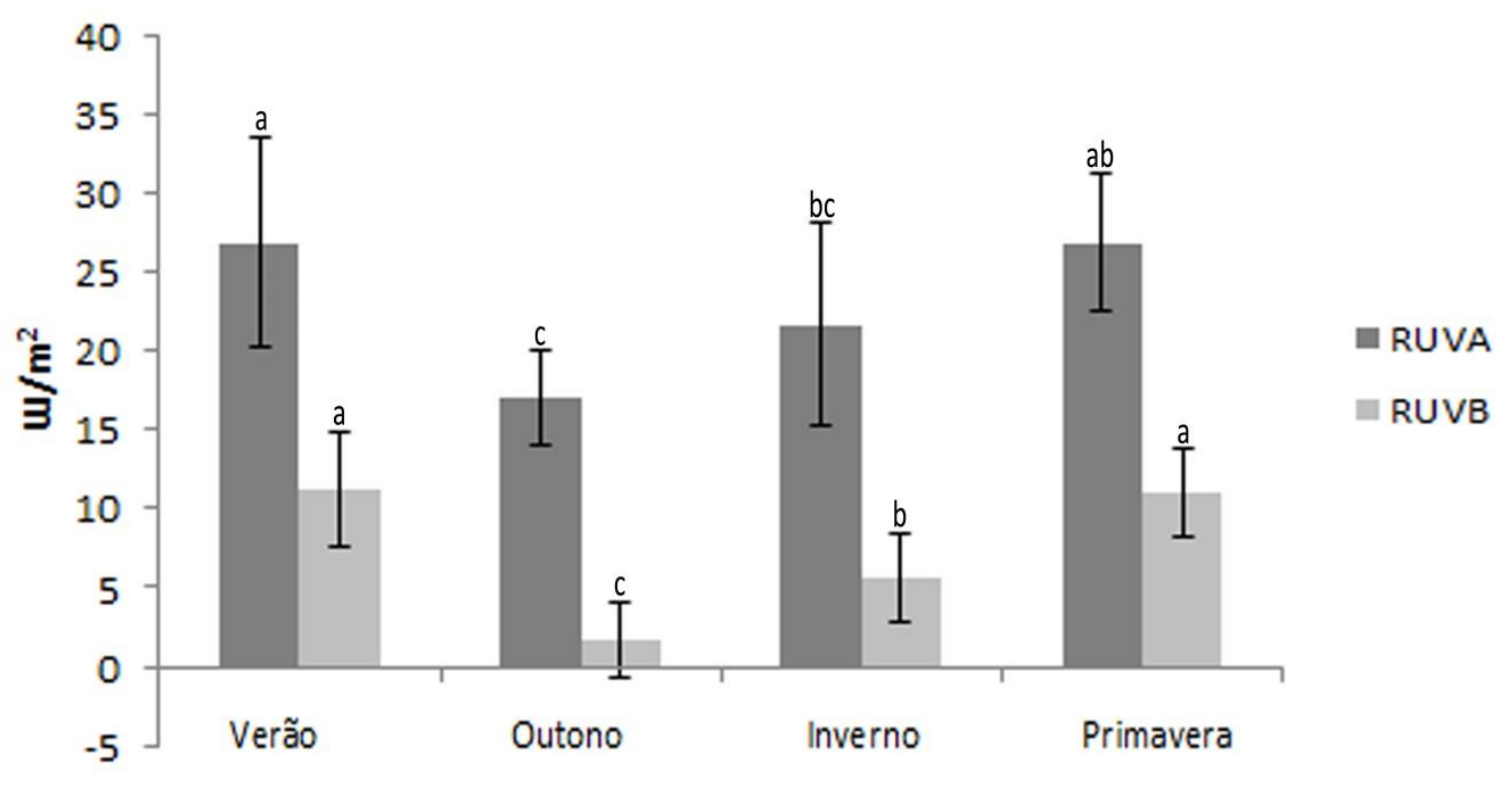

Fig. 6 Medidas sazonais da intensidade de RUVA e RUVB $\left(\mathrm{U} / \mathrm{m}^{2}\right)$. Maiores índices de RUVA e RUVB ocorreram no verão, seguido da primavera, enquanto o outono foi a estação com menores índices de RUVA e RUVB.
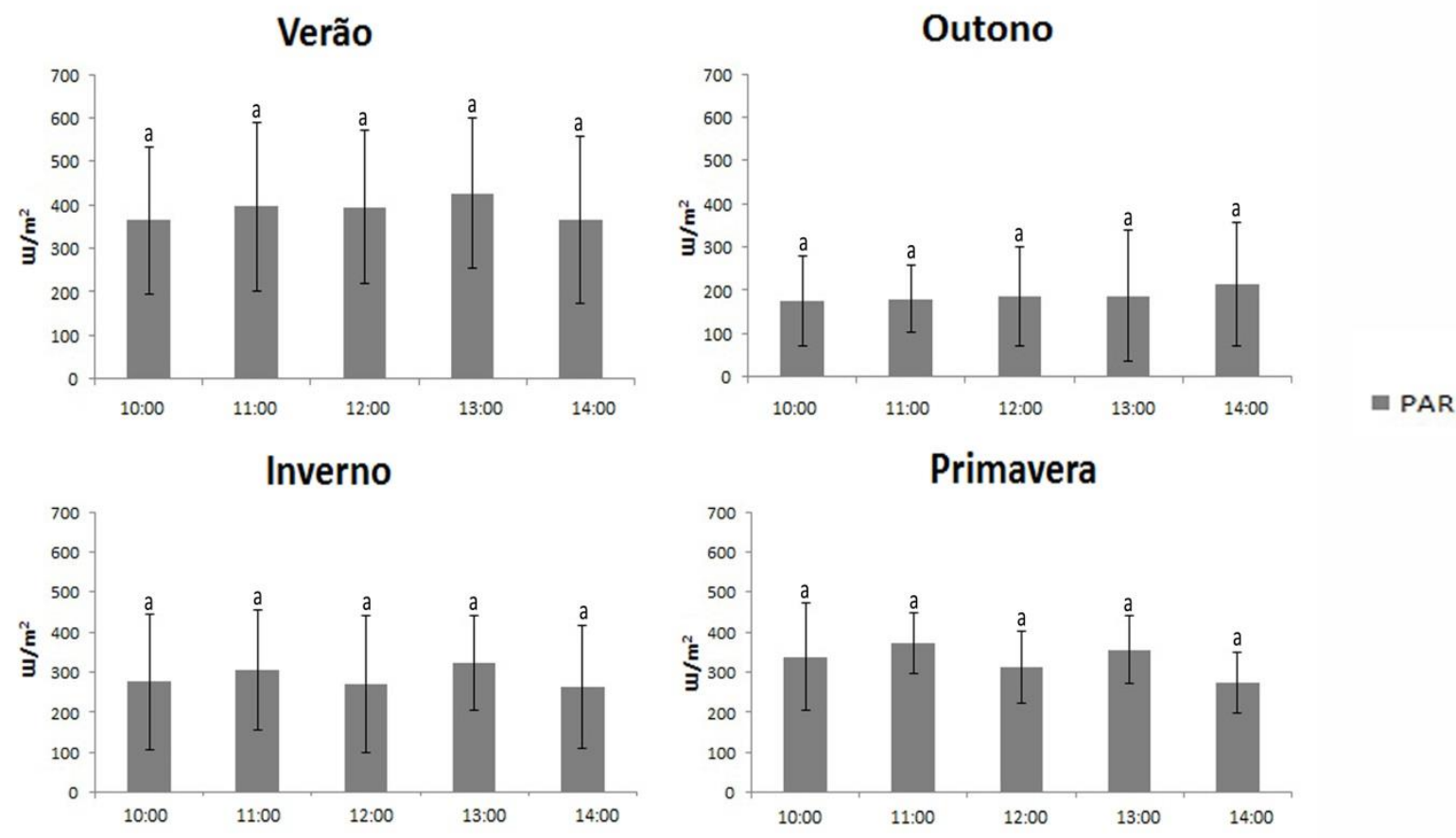

Fig. 7 Intensidade de PAR $\left(\mathrm{W} / \mathrm{m}^{2}\right)$ durante as estações e horários (10:00, 11:00, 12:00, 13:00 e 14:00). Dentro de cada estação, não se observou diferença estatística da intensidade da PAR entre os horários medidos. 

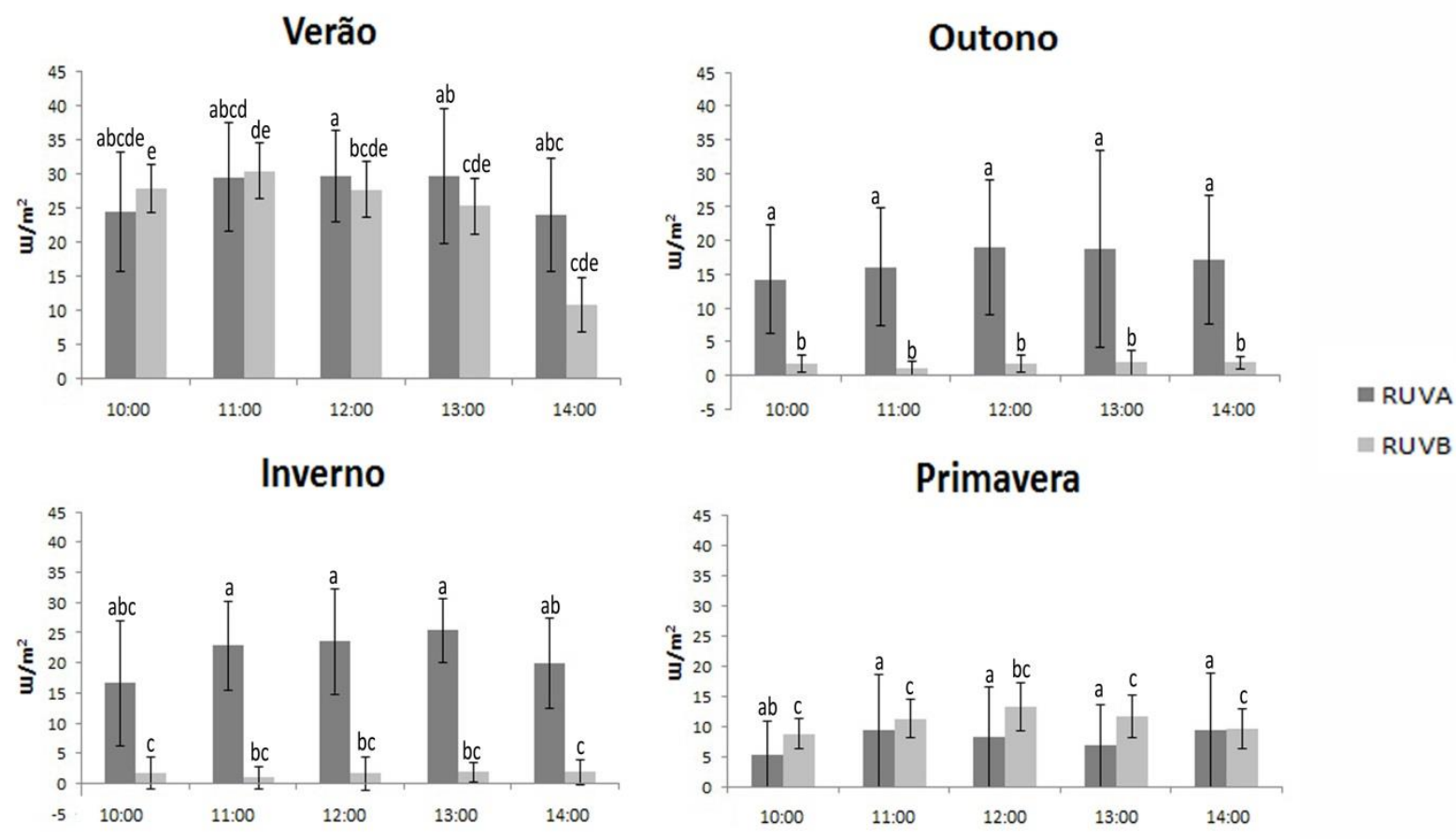

Fig. 8 Intensidade de RUVA e RUVB $\left(\mathrm{W} / \mathrm{m}^{2}\right)$ durante as estações e horários (10:00, 11:00, 12:00, 13:00 e 14:00). Tanto a RUVA e a RUVB apresentaram maiores intensidades no verão e ao meio dia, enquanto nas outras estações não houve diferença estatística entre as intensidades e os horários medidos.

\section{Medidas por Horário}

Ao longo do ano, observou-se que as maiores intensidades de PAR ocorreram às 11:00 e 13:00, enquanto que para RUVA e RUVB ocorreram às 12:00 (Fig. 9). 

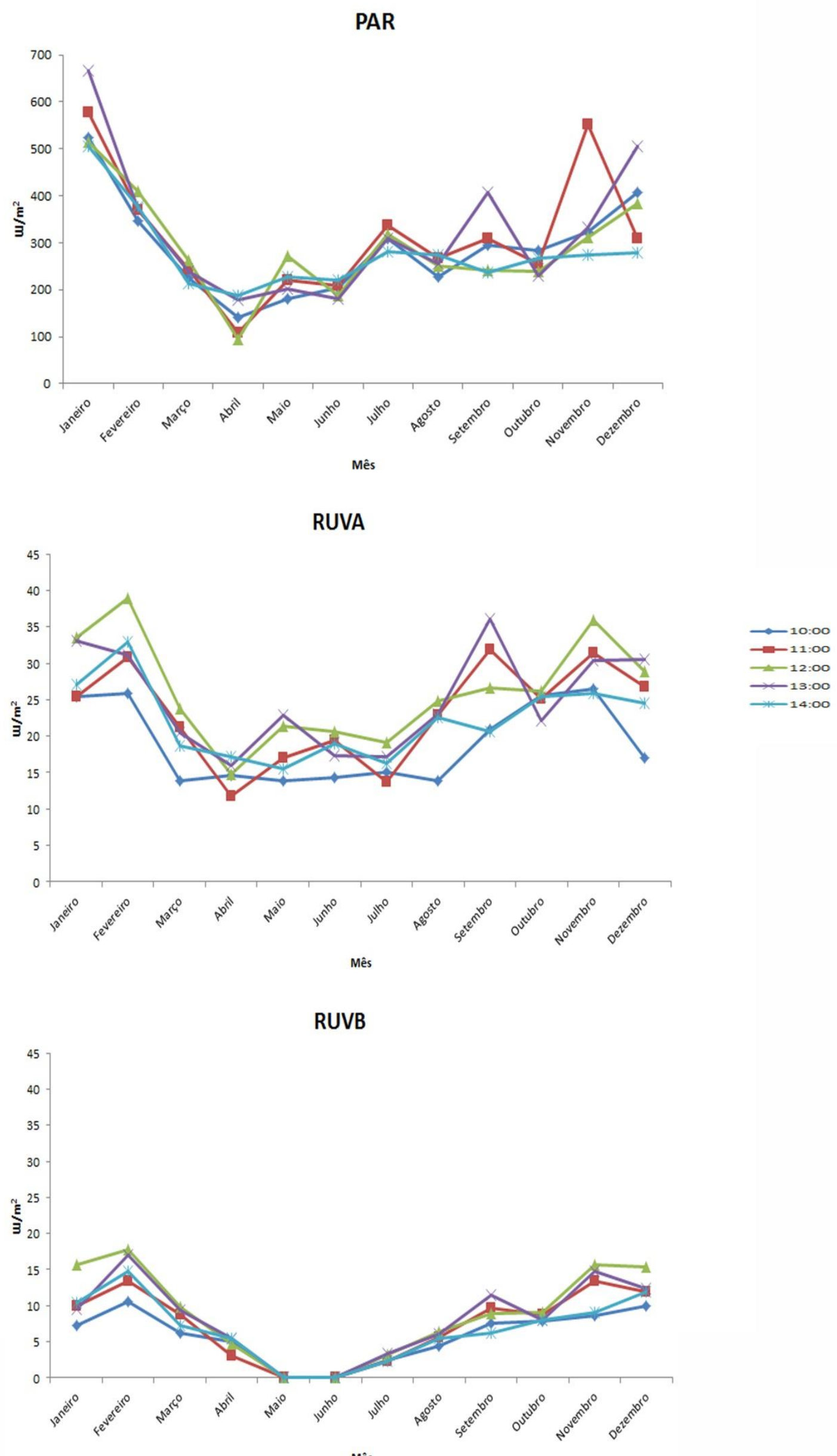
Fig. 9 Intensidades de PAR, RUVA e RUVB $\left(\mathrm{W} / \mathrm{m}^{2}\right)$ durante os meses do ano e horários $(10: 00,11: 00,12: 00,13: 00$ e 14:00). Observou-se que as maiores intensidades de PAR ocorreram às 11:00 e 13:00, enquanto que para RUVA e RUVB ocorreram às 12:00.

\section{Discussão}

A PAR é a radiação conhecida como o espectro solar utilizado na conversão de energia luminosa em biomassa, ou seja, a radiação utilizada na fotossíntese (JACOVIDES et al., 2010; SADRAS et al., 2017; STAMENKOVIC; HALNET 2017). Além disso, a PAR é importante em várias aplicações relacionadas à fisiologia das plantas, produção de biomassa, iluminação natural em estufas e pesquisa agrícola (JACOVIDES et al., 2010). Medidas de PAR na Lagoa do Peri, em Florianópolis (SCBrasil) indicaram que, na superfície da água, chega até $250 \mu \mathrm{mol} \mathrm{m} \mathrm{m}^{2} \mathrm{~s}$, o que equivale a $50 \mathrm{~m} / \mathrm{m}^{2}$ (TONETTA, 2016). O local de registro das informações do presente estudo (UFSC-Florianópolis) está localizado na mesma cidade da Lagoa do Peri, a intensidade desta radiação chega a até $600 \mathrm{~m} / \mathrm{m}^{2}$, principalmente nos meses de janeiro e dezembro, período em que ocorre o solstício de verão. Para os outros meses a média da PAR recebida foi de $250 \mathrm{~m} / \mathrm{m}^{2}$. Percebemos uma menor intensidade de PAR na Lagoa do Peri, muito provavelmente pela quantidade de árvores, que exercem sombra, e maior umidade, favorecendo a formação de nuvens, as quais barram as radiações.

Para as plantas e algas, a RUVA é conhecida por ser benéfica, auxiliando no crescimento (POLO et al., 2014), no desenvolvimento (HENRY; ALSTYNE 2004) e na produção da enzima RuBisCo (BISCHOF et al., 2002). Já a RUVB é conhecida por ser prejudicial, causando efeitos negativos sobre as plantas, tanto nos seus processos fisiológicos, bioquímicos, morfológicos e moleculares (BISCHOF et al., 2000; WIENCKE et al., 2000; HEO et al., 2010; ROLEDA et al., 2010; SCHMIDT et al., 2010; FREITAS, 2012; FIGUEROA et al., 2014; PEREIRA et al., 2014; POLO et al., 2014). Por outro lado, para o ser humano, a exposição à RUVA e RUVB traz benefícios à saúde, já que está relacionada à síntese de vitamina $\mathrm{D}$ e prevenção de doenças como osteoporose, diabetes tipo 1, alguns tipos de câncer e doenças autoimunes (CORREA, 2015). Porém, a exposição à altas intensidades de RUVA e RUVB podem causar distúrbios oculares, como catarata e pterígio, bem como distúrbios da pele, entre eles queimaduras solares, envelhecimento prematuro e câncer (CORREA, 2015; MELO et al., 2015). 
KIRCHHOFF et al., (2000) investigaram a intensidade de RUVB em algumas cidades da América do Sul entre os anos de 1994 a 1999. Durante este período as maiores intensidades de RUVB estão ditas a seguir: Punta Arenas (Chile) máximo de 1,10 $\mathrm{m} / \mathrm{m}^{2}$; Blumenau (SC-Brasil) e Porto Alegre (RS-Brasil) máximo de 1,3 $\mathrm{U} / \mathrm{m}^{2}$; Cachoeira Paulista (SP-Brasil), Campo Grande (MS-Brasil) e Ribeirão Preto (SP-Brasil) máximo de 1,60 $\mathrm{m} / \mathrm{m}^{2}$; Natal (RN-Brasil) máximo de 1,70 $\mathrm{m} / \mathrm{m}^{2}$; La Paz (Bolívia) máximo de 2,10 $\mathrm{m} / \mathrm{m}^{2}$. Sabe-se que em menores latitudes, mais intensa é a radiação que chega na superfície terrestre, como observado em KIRCHHOFF et al., (2000), GUARNIERI et al., (2004) e PINTO et al., (2010). Porém, o efeito da altitude também influencia nesta intensidade de radiação recebida, como observado em La Paz que superou Campo Grande, ambas na mesma latitude, porém La Paz com altitude maior. Em maiores altitudes a troposfera deve ser mais rarefeita, facilitando a passagem da RUVB. O bairro Trindade em Florianópolis, onde ocorreu a pesquisa, possui em torno de 3 metros de altitude, esta altitude é mais baixa quando comparada com todas as outras cidades citadas anteriormente acima, porém mesmo com baixa altitude apresentou elevadas incidências de RUVB, com uma máxima de $17 \mathrm{~m} / \mathrm{m}^{2}$. Com estes dados podemos comparar e perceber que mesmo sendo uma cidade mais baixa, em relação ao nível o mar, o que normalmente implicaria em menor incidência de RUVB, que com o passar de 14 anos a cidade se desenvolveu e com este desenvolvimento a quantidade de gases poluentes que vão para atmosfera e que destroem a camada de $\mathrm{O}_{3}$ aumentaram, o que pode explicar este aumento considerável de RUVB. O mesmo ocorreu em São Paulo (SP-Brasil), onde medidas de RUVB tiveram um aumento com o passar de 8 anos de análises (CORREA, 2015).

Entre 2002 a 2004 foram medidas as intensidades de RUVA em São Martinho da Serra (RS-Brasil) e Tóquio (Japão), e percebeu-se que RUVA chegava até 70 $\mathrm{U} / \mathrm{m}^{2}$ em São Martinho da Serra, enquanto que no Japão chegava a $55 \mathrm{~m} / \mathrm{m}^{2}$ (NOZAWA et al. 2007). As medidas realizadas em Florianópolis (SC-Brasil) apresentaram uma intensidade máxima de $40 \mathrm{~m} / \mathrm{m}^{2}$ de RUVA, ou seja, intensidade bem mais baixa de RUVA quando comparado com São Martinho da Serra e Tóquio. Esta diferença na intensidade de RUVA pode ser devido a altitude de cada lugar, Florianópolis (UFSC) fica a 3 metros, Tóquio a 40 metros enquanto São Martinho fica a 450 metros. Como visto anteriormente, locais em maiores altitudes apresentam maior facilidade na chegada de radiação, visto que a atmosfera é mais rarefeita, e os raios não encontram muitas barreiras (moléculas) para serem 
atenuados e dissipados, implicando em maiores intensidades de radiações. Como a RUVA tem maior comprimento de onda, porém menor energia, ela é pouco atenuada pelos gases atmosféricos, atingindo com maior facilidade a superfície terrestre.

Em um levantamento dos últimos estudos publicados que trabalham com PAR, RUVA e RUVB temos as seguintes intensidades utilizadas: um estudo dos efeitos sobre a viscosidade dinâmica das soluções de riboflavina à base de gelatina na Turquia, utilizando $6 \mathrm{~mm} / \mathrm{cm}^{2}$ de RUVA (equivalente a 0,006 $\mathrm{m} / \mathrm{m}^{2}$ ) (DEMIRBAY, 2016); o estudo com a diatomácea Thalassiosira (Conticribra) weissflogii na China usou intensidade de 53 a $409 \mathrm{~m} / \mathrm{m}^{2}$ de PAR, de 10,38 a 72,08 $\mathrm{m} / \mathrm{m}^{2}$ de RUVA e de 0,28 a $1,90 \mathrm{~m} / \mathrm{m}^{2}$ de RUVB (LI et al. 2016); um estudo com o dinoflagelado Symbiodinum, na ilha de Hainan (China), usou $1000 \mu \mathrm{mol} \mathrm{m}{ }^{2} / \mathrm{s}$ (equivalente a 200 $\mathrm{m} / \mathrm{m}^{2}$ ) de PAR, $50 \mathrm{~m} / \mathrm{m}^{2}$ de RUVA e $25 \mathrm{~m} / \mathrm{m}^{2}$ de RUVB (ZHOU et al. 2017); um estudo com Cecropia obtusa em Bélem (PA-Brasil) usou RUVA por 24 horas gerando uma dose de $4 \mathrm{~J} / \mathrm{cm}^{2}$ (equivalente a $0,000046 \mathrm{~m} / \mathrm{m}^{2}$ ) e RUVB com uma dose de $0,02 \mathrm{~J} / \mathrm{cm}^{2}$ (equivalente a $0,000002 \mathrm{~m} / \mathrm{m}^{2}$ ) (ALVES et al. 2016); estudo em São Paulo (SP-Brasil) com fungos Metarhizium anisopliae (Metsch.) Sorok. e Beauveria bassiana (Bals.) Vuill. foram irradiados com 680, 750, 780 e $800 \mathrm{~m} / \mathrm{m}^{2} \mathrm{de}$ PAR e $12 \mathrm{mU} / \mathrm{cm}^{2}$ (equivalente a 0,012 $\mathrm{m} / \mathrm{m}^{2}$ ) de RUVB (OLIVEIRA et al. 2016); estudo com a árvore frutífera Dillenia indica Linn. (Dilleniaceae) em Tubarão (SCBrasil) usou $0.00083 \mathrm{~m} / \mathrm{m}^{2}$ de RUVB (KVIECINSKI, 2016); um estudo com a macroalga Sargassum cymosum em Florianópolis (SC-Brasil) utilizou $80 \mu \mathrm{mol} \mathrm{m} / \mathrm{s}$ (equivalente a $16 \mathrm{~m} / \mathrm{m}^{2}$ ) de PAR (COSTA et al. 2016); e um estudo com camarão Macrobrachium olfersi em Florianópolis (SC-Brasil) usou $310 \mathrm{~mm} / \mathrm{cm}^{2}$ (equivalente a $0,31 \mathrm{~m} / \mathrm{m}^{2}$ ) de RUVB (QUADROS et al., 2016).

Com estes dados, percebemos que os estudos em laboratórios trabalham com intensidades de PAR e RUVA perto da realidade ambiental, porém maiores intensidades destas radiações também estariam dentro do normal. Por outro lado, percebemos baixas intensidades e doses de RUVB quando comparados com os resultados obtidos no presente trabalho, com exceção do trabalho mais recente (ZHOU et al., 2017) que traz altos níveis tanto de RUVA e RUVB, se adequando mais a realidade. Sabe-se que em um experimento em laboratório torna-se limitado, pois precisa-se isolar apenas algumas variáveis, aquelas que se quer estudar, não levando em conta todas as interações de variáveis que se pode ter no meio ambiente. Portanto, o motivo de menores intensidades verificadas nos trabalhos, tanto para PAR, RUVA e RUVB, pode ser devido a esta limitação do experimento. 
Por outro lado, estas intensidades baixas, com relação ao ambiente, já trazem respostas muito interessantes e drásticas, mostrando que mesmo em baixas doses destas radiações já temos um efeito biológico. Os principais efeitos observados, mesmo com doses baixas de RUVB, foram: queda da taxa de crescimento e de germinação, formação de espécies reativas de oxigênio (moléculas tóxicas ao metabolismo), alterações estruturais e funcionais de organelas, entre outros.

Por fim, conclui-se que as intensidades de PAR e RUVB estão aumentadas no bairro Trindade, Florianópolis, quando comparamos com outros locais, enquanto que a RUVA se mantém no esperado. Como a região em questão está poucos metros acima do nível do mar, estes resultados podem nos ajudar na compreensão de como o meio ambiente está reagindo ao crescimento de Florianópolis, pois quanto maiores as intensidades destas radiações, maiores as degradações da camada de $\mathrm{O}_{3}$. Estes dados tornam-se públicos e podem abrir portas para novos estudos com relação a poluição do local, e de estratégias ambientais a fim de buscar melhores resultados para a comunidade.

\section{REFERÊNCIAS}

ANDERSON, L. E. et al. Non-ionizing radiation, Part 1, static and extremely low-frequency (ELF) electric and magnetic fields. IARC monographs on the evaluation of carcinogenic risks to humans, v. 80, p. 43-46, 2002.

ALVES, G. A. D. et al. Cecropia obtusa, an Amazonian ethanolic extract, exhibits photochemoprotective effect in vitro and balances the redox cellular state in response to UV radiation. Industrial Crops and Products, v. 94, p. 893-902, 2016.

BALOGH, T. S. et al. Proteção à radiação ultravioleta: recursos disponíveis na atualidade em fotoproteção. Anais Brasileiros de Dermatologia, v. 86(4), p. 732-742, 2011.

BEISSERT, S.; GRANSTEIN, R. D. UV-induced cutaneous photobiology. Critical Reviews in Biochemistry and Molecular Biology, v. 31, p. 381-404, 1996.

BISCHOF, K. et al. Effects of solar UV-B radiation on canopy structure of Ulva communities from southern Spain. Journal of Experimental Botany, v. 53(379), p. 2411-2421, 2002. BISCHOF, K.; HANELT, D.; WIENCKE, C. Effects of ultraviolet radiation on photosynthesis and related enzyme reactions of marine macroalgae. Planta, v. 211, p. 555-562, 2000. CASSETI, V.; COSTA, J. E. A. M. Algumas considerações dobre o ozônio e o projeto ozônio em Goiás. Boletim Goiano de Geografia, v. 9, p. 21-42, 1989.

CORRÊA, M. P. Solar ultraviolet radiation: properties, characteristics and amounts observed in Brazil and South America. Anais Brasileiros de Dermatologia, v. 90, p. 297-313, 2015. COSTA, G. B. et al. The brown seaweed Sargassum cymosum: changes in metabolism and cellular organization after long-term exposure to cadmium. Protoplasma, v. 254(2), p. 817837, 2016.

DE LAAT, A et al. Cell cycle kinetics following UVA irradiation in comparison to UVB and UVC irradiation. Photochemistry and Photobiology, v. 63, p. 492-7, 1996.

DE QUADROS, T. et al. Developmental effects of exposure to ultraviolet $B$ radiation on the freshwater prawn Macrobrachium olfersi: Mitochondria as a target of environmental UVB radiation. Ecotoxicology and Environmental Safety, v. 132, p. 279-287, 2016.

DEMIRBAY, B. et al. Thermal and UV radiation effects on dynamic viscosity of gelatin-based riboflavin solutions. Journal of Molecular Liquids, v. 225, p. 147-150, 2017. 
DIFFEY, B. L. Solar ultraviolet radiation effects on biological systems. Physics in Medicine and Biology, v. 36, p. 299-328, 1991.

DIFFEY, B. L. Sources and measurement of ultraviolet radiation. Methods, v. 28, p. 4-13, 2002.

ELLI, E. F. et al. Ecofisiologia da cana-de-açúcar no sub-bosque de canafístula em arranjos de sistema agroflorestal. Comunicata Scientiae, v. 7, p. 464, 2017.

ESCOBEDO, J. F. et al. Ratios of UV, PAR and NIR components to global solar radiation measured at Botucatu site in Brazil. Renewable Energy, v. 36, p. 169-178, 2011.

FIGUEROA, F. L. et al. Short-term effects of increasing $\mathrm{CO}_{2}$, nitrate and temperature on three mediterranean macroalgae: Biochemical composition. Aquatic Biology, v. 22, p. 177193, 2014.

FREITAS, M. Influência de fatores exógenos na germinação de esporos e da radiação ultravioleta B (RUVB) no desenvolvimento de gametófitos de Acrostichum danaeifolium Langsd. \& Fisch. (Polypodiopsida, Pteridaceae): uma pteridófita do manguezal. Dissertação da Universidade Federal de Santa Catarina, 2012.

GUARNIERI, R. A. et al. Ozone and UV-B radiation anticorrelations at fixed solar zenith angles in southern Brazil. Geofisica Internacional, v. 43, p. 17-22, 2004.

HENRY, B. E.; VAN ALSTYNE, K. L. Effects of UV radiation on growth and phlorotannins in Fucus gardneri (Phaeophyceae) juveniles and embryos. Journal of Phycology, v. 40(3), p. 527-533, 2004.

HEO, S. et al. Inhibitory effect of diphlorethohydroxycarmalol on melanogenesis and its protective effect against UV-B radiation-induced cell damage. Food and Chemical Toxicology, v. 48, p. 1355-1361, 2010.

HOLLÓSY, F. Effects of ultraviolet radiation on plant cells. Micron, v. 33, p. 179-197, 2002. JACOVIDES, C. P. et al. Comparing diffuse radiation models with one predictor for partitioning incident PAR radiation into its diffuse component in the eastern Mediterranean basin. Renewable Energy, v. 35, p. 1820-1827, 2010.

KIRCHHOFF, V. W. J. H. Geoquímica da média e baixa atmosfera: impactos ambientais por deterioração da camada de ozônio. Geochimica Brasiliensis, v. 2(1), p. 41-52, 1988.

KIRCHHOFF, V. W. J. H. et al. A variação sazonal da radiação ultravioleta solar biologicamente ativa. Revista Brasileira de Geofisica, v. 18, p. 62-73, 2000.

KVIECINSKI, M. et al. Healing effect of Dillenia indica fruit extracts standardized to betulinic acid on ultraviolet radiation-induced psoriasis-like wounds in rats. Pharmaceutical Biology, v. 55, p. 641-648, 2016.

$\mathrm{LI}, \mathrm{W}$. et al. Effects of seawater acidification on the growth rates of the diatom Thalassiosira (Conticribra) weissflogii under different nutrient, light, and UV radiation regimes. Journal of Applied Phycology, p. 1-10, 2016.

ELO, M. M.; CARVALHO, C. S. DE. Novas considerações sobre a fotoproteção no Brasil:

Revisão de literatura. Revista Ciências em Saúde, v. 5(3), p. 1-17, 2015.

MOLINA, L. T.; MOLINA, M. J. Production of $\mathrm{Cl}_{2} \mathrm{O}_{2}$ from the self-reaction of the $\mathrm{ClO}$ radical. The Journal of Physical Chemistry, v. 91, p. 433-436, 1987.

MOLINA, M. J.; ROWLAND, F. S. Stratospheric sink for chlorofluoromethanes: chlorine atomc-atalysed destruction of ozone. Nature, v. 249, p. 810-812, 1974.

NOZAWA, H. et al. Ground-based observations of solar UV radiation in Japan, Brazil and Chile. Revista Brasileira de Geofísica, v. 25 Supl., p. 17-25, 2007.

OKUNO, E. et al. Radiação ultravioleta solar em São Paulo, China, Calafate e llha de Páscoa. Caderno de Engenharia Biomédica, v. 12(3), p. 143-153, 1996.

OLIVEIRA, M. T. DE et al. Sensibilidade de isolados de fungos entomopatogênicos às radiações solar, ultravioleta e à temperatura. Arquivos do Instituto Biológico, v. 83, p. 17, 2016.

PEREIRA, D. T. et al. The effects of ultraviolet radiation-B response on the morphology, ultrastructure, and photosynthetic pigments of Laurencia catarinensis and Palisada flagellifera (Ceramiales, Rhodophyta): a comparative study. Journal of Applied Phycology, v. 26, p. 2443-2452, 2014.

PINTO, L. I. C. et al. Comparação de produtos de radiação solar incidente à superfície para a América do Sul. Revista Brasileira de Meteorologia, v. 25, p. 469-478, 2010. POLO, L. K. et al. Photoacclimation responses of the brown macroalga Sargassum 
cymosum to the combined influence of UV radiation and salinity: Cytochemical and ultrastructural organization and photosynthetic performance. Photochemistry and Photobiology, v. 90, p. 560-573, 2014.

ROLEDA, M. Y. et al. Physiological , biochemical , and ultrastructural responses of the green macroalga Urospora penicilliformis from Arctic Spitsbergen to UV radiation. Protoplasma, v. 243, p. 105-116, 2010.

SCHARFFETTER-KOCHANEK, K. et al. UV-induced reactive oxygen species in photocarcinogenesis and photoaging. Biological chemistry, v. 378, p. 1247-1257, 1997. SCHMIDT, É. C. et al. Effect of ultraviolet-B radiation on growth, photosynthetic pigments , and cell biology of Kappaphycus alvarezii (Rhodophyta, Gigartinales) macroalgae brown strain. Photosynthetica, v. 48(2), p. 161-172, 2010.

STAMENKOVIC, M.; HANELT, D. Geographic distribution and ecophysiological adaptations of desmids (Zygnematophyceae, Streptophyta) in relation to PAR, UV radiation and temperature: a review. Hydrobiologia, v. 787, p. 1-26, 2016.

STOLARSKI, S.; CICERONE, J. Stratospheric chlorine: a possible sink for ozone.

Cannadian Journal Chemistry, v. 258, p. 30-38, 1974.

TONETTA, D. et al. Physical conditions driving the spatial and temporal variability in aquatic metabolism of a subtropical coastal lake. Limnologica, v. 58, p. 30-40, 2016.

WIENCKE, C. et al. Impact of UV-radiation on viability, photosynthetic characteristics and DNA of brown algal zoospores: implications for depth zonation. Marine Ecology Progress Series, v. 197, p. 217-229, 2000.

ZHOU, J. et al. Effect of UV radiation on the expulsion of Symbiodinium from the coral Pocillopora damicornis. Journal of Photochemistry and Photobiology B: Biology, v. 166, p. 12-17, 2017.

\section{NOTAS DE AUTOR}

\section{CONTRIBUIÇÃO DE AUTORIA}

Débora Tomazi Pereira - Concepção, coleta de dados, análise de dados, elaboração do manuscrito, revisão e aprovação da versão final do trabalho

Zenilda Laurita Bouzon - Concepção, análise de dados, revisão e aprovação da versão final do trabalho Carmen Simioni - Concepção, análise de dados, elaboração do manuscrito, revisão e aprovação da versão final do trabalho

\section{FINANCIAMENTO}

Não se aplica.

\section{CONSENTIMENTO DE USO DE IMAGEM}

Não se aplica.

\section{APROVAÇÃO DE COMITÊ DE ÉTICA EM PESQUISA \\ Não se aplica.}

\section{CONFLITO DE INTERESSES}

Não se aplica.

\section{LICENÇA DE USO}

Este artigo está licenciado sob a Licença Creative Commons CC-BY. Com essa licença você pode compartilhar, adaptar, criar para qualquer fim, desde que atribua a autoria da obra.

\section{HISTÓRICO}

Recebido em: 23-11-2017

Aprovado em: 03-08-2019 